\title{
Minimally invasive adrenalectomy: a comprehensive systematic review and network meta-analysis of phase II/III randomized clinical controlled trials
}

\author{
Laura Alberici ${ }^{1}$. Carlo Ingaldi ${ }^{1,2}$. Claudio Ricci ${ }^{1,2,3}$. Saverio Selva ${ }^{1}$. Guido Di Dalmazi ${ }^{2,4}$. Valentina Vicennati ${ }^{2,4}$. \\ Uberto Pagotto $^{2,4} \cdot$ Riccardo Casadei $^{1,2} \cdot$ Francesco Minni $^{1,2}$
}

Received: 22 October 2021 / Accepted: 2 January 2022 / Published online: 12 January 2022

(C) The Author(s) 2022

\begin{abstract}
Purpose The best approach for minimally invasive adrenalectomy is still under debate.

Methods A systematic search of randomized clinical trials was carried out. A frequentist random-effects network metaanalysis was made reporting the surface under the cumulative ranking (SUCRA). The primary endpoint regarded both inhospital mortality and morbidity. The secondary endpoints were operative time (OP), blood loss (BL), length of stay (LOS), conversion, incisional hernia, and disease recurrence rate.

Results Eight studies were included, involving 359 patients clustered as follows: 175 (48.7\%) in the TPLA arm; 55 (15.3\%) in the RPLA arm; 10 (2.8\%) in the Ro-TPLA arm; 25 (7\%) in the TPAA arm; 20 (5.6\%) in the SILS-LA arm; and 74 (20.6\%) in the RPA arm. The RPLA had the highest probability of being the safest approach (SUCRA 69.6\%), followed by RPA (SUCRA 63.0\%). TPAA, Ro-TPLA, SILS-LA, and TPLA have similar probability of being safe (SUCRA values $45.2 \%$, $43.4 \%, 43.0 \%$, and $38.5 \%$, respectively). Analysis of the secondary endpoints confirmed the superiority of RPA regarding $\mathrm{OP}, \mathrm{BL}, \mathrm{LOS}$, and incisional hernia rate.

Conclusions The best choice for patients with adrenal masses candidate for minimally invasive surgery seems to be RPA. An alternative could be RPLA. The remaining approaches could have some specific advantages but do not represent the first minimally invasive choice.
\end{abstract}

Keywords Laparoscopic adrenalectomy $\cdot$ Retroperitoneal adrenalectomy $\cdot$ Network meta-analysis $\cdot$ Safety $\cdot$ Efficacy

Claudio Ricci

claudiochir@gmail.com

1 Division of Pancreatic Surgery, IRCCS Azienda Ospedaliero-Universitaria Di Bologna, via Albertoni 15, Bologna, Italia

2 Department of Internal Medicine and Surgery, DIMEC) Alma Mater Studiorum, University of Bologna, S.Orsola-Malpighi Hospital, Bologna, Italy

3 Dipartimento Di Scienze Mediche E Chirurgiche (DIMEC), Chirurgia Generale-Minni, Alma Mater Studiorum-Università Di Bologna, IRCCS, Policlinico S.Orsola-Malpighi, Via Massarenti n.9 40138, Bologna, Italy

4 Unit of Endocrinology and Diabetes Prevention and Care, IRCCS Azienda Ospedaliero-Universitaria Di Bologna, via Albertoni 15, Bologna, Italia

\section{Introduction}

Over the last 30 years, the minimally invasive approach to adrenalectomy has almost replaced open surgery in the management of most adrenal pathologies and tumors. Since 1992, year in which for the first time in literature Gagner [1] reported three cases of transperitoneal laparoscopic adrenalectomy performed by lateral approach with the patient in lateral decubitus, other surgical minimally invasive approaches to the adrenal glands had been introduced. One of these is the transperitoneal laparoscopic adrenalectomy performed by anterior approach with the patient in supine position, as reported by Lezoche et al [2] in a large series of cases in the late 1990s. Completely different approaches are the posterior retroperitoneoscopic adrenalectomy, with the patient in prone position, first described by Mercan [3], but later advanced and popularized by Walz [4], and the retroperitoneoscopic adrenalectomy with the patient in lateral 
decubitus, reported by Bonjer [5]. More recent minimally invasive techniques include the single-incision adrenalectomy for both laparoscopic or retroperitoneoscopic adrenalectomy and the robotic approach. Gaining experience in one or the other technique, many surgeons reported their cases in retrospective observational studies, sometimes testing their learning curve, other times comparing their results with those obtained with different techniques. All minimally invasive approaches have demonstrated their feasibility and safety. However, according to trials published so far, there is no consensus regarding which approach is the most suitable or has significant advantages over the others. Only a few meta-analyses are available, but data coming from prospective trials and comparative studies retrospective in nature are often mixed with each other creating concern about the accuracy and reliability of the analysis itself. Remarkably, any of the meta-analyses currently published in the literature reviewed only randomized controlled trials, which represent studies with the highest level of evidence. Moreover, metaanalyses constitute an important source of evidence-based practice but do have a significant drawback: they can compare only 2 interventions simultaneously.

Therefore, we chose to perform a network meta-analysis (NMA). This statistical evaluation preserves the randomized nature of data and has the advantage of assessing the effectiveness of several different approaches through the combination of both direct (head-to-head) and indirect (through common comparators) evidence to gain certainty about all treatment comparisons.

Our study aimed to define the safety and efficacy of the different approaches to adrenalectomy.

\section{Methods}

A systematic review was performed according to the Cochrane recommendations [6], and the paper was structured following the PRISMA checklist (Preferred Reporting Items for Systematic Reviews and Meta-Analyses) [7]. The approval by an institutional review board was not required.

\section{Eligibility criteria}

The eligibility criteria were established according to PICOS criteria [8]:

1) "Population": the "Population" was represented by the patients diagnosed with resectable benign or malignant adrenal tumor candidates to minimally invasive adrenalectomy (MIA)

2) "Intervention": the "Intervention" arms were all minimally invasive approaches different from transperitoneal laparoscopic lateral adrenalectomy
3) "Control": the "Control" group was the transperitoneal laparoscopic lateral adrenalectomy

4) "Outcomes": all studies reporting at least the postoperative morbidity and length of postoperative stay (LOS) were included

5) "Studies": all phase II/III RCTs, including at least two arms

NMA approach was used to avoid the problem derived from a multi-arm setting $[9,10]$. The intervention arms were clustered according to three main parameters: the type of approach (transperitoneal or retroperitoneal), the surgical access (anterior, lateral, or posterior), and the application of particular devices for minimally invasive surgery (single incision or robotic approach). Thus, six intervention arms were planned: transperitoneal laparoscopic lateral adrenalectomy (TPLA); retroperitoneal minimally invasive lateral adrenalectomy (RPLA); transperitoneal laparoscopic lateral adrenalectomy with robotic approach (Ro-TPLA); transperitoneal laparoscopic anterior adrenalectomy (TPAA); singleincision laparoscopic adrenalectomy (SILS-LA); retroperitoneal minimally invasive posterior adrenalectomy (RPA). The TPLA was used as a referent arm. No other technical differences were used to additionally divide the surgical procedures to avoid an excessive scattering of the network.

\section{Information source, search, study selection, and data collection process}

The information sources were MEDLINE (via PubMed), Web of Science, and Scopus, and the last search was carried out on August 7, 2021. The studies' bibliography was included for additional reference. All eligible studies were read in full-text form by two independent investigators (G.D. and L.A.). When the inclusion criteria were fulfilled in the absence of exclusion criteria, the paper was included. Two independent reviewers (C.R. and C.I.) carried out data extraction using a dedicated form. Any disagreement between the reviewers was solved by a collegial discussion with the senior author (F.M.). A PRISMA flowchart was created to show the authors' conclusions (Fig. 1). Additional details regarding the eligibility criteria, information sources, search, study selection, and data collection process are exhaustively reported in a Supplementary file (S_Method).

\section{Data items}

The primary endpoint was a composite endpoint, including the analysis of postoperative mortality and complications. The choice of using a composite endpoint was due to the low mortality rate that characterized the adrenal surgery, hence the need to group together mortality and complications as indicators of safety. The secondary 


\section{Flow Diagram}

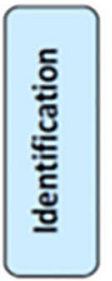

Records identified through Medline/PubMed $(n=3508)$,

Scopus $(n=4643)$, ISI web of science search $(n=1636)$

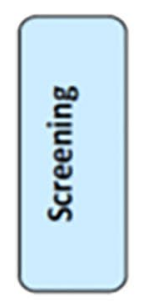

Records after duplicates removed

( $n=4643$ )
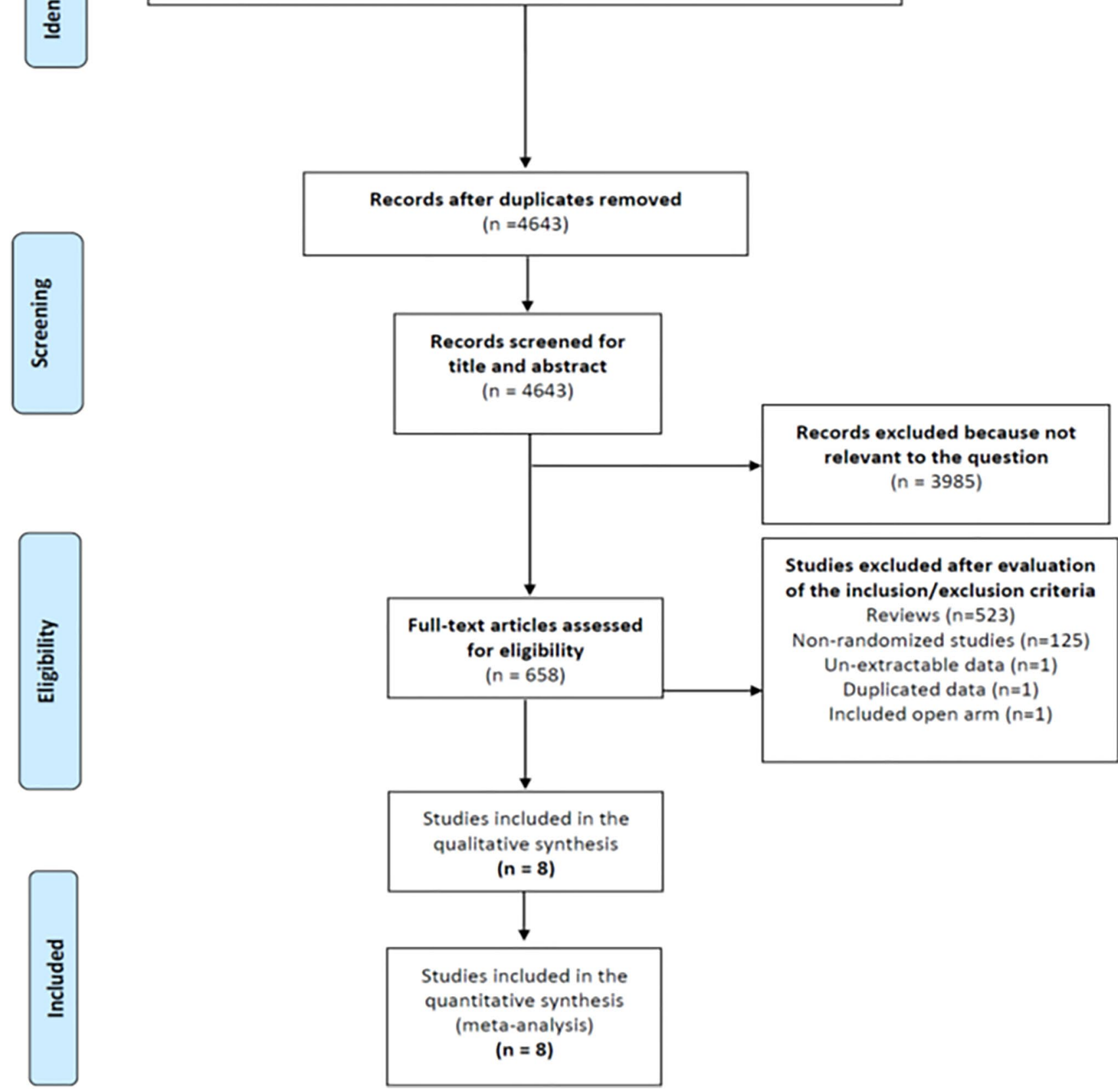

Fig. 1 PRISMA flowchart. PRISMA = Preferred Reporting Items for Systematic Reviews and Meta-Analyses

endpoints were the analysis of operative time, blood loss, length of stay (LOS), conversion rate, incisional hernia, and disease recurrence rate. A safety/efficacy ratio was obtained using the composite endpoint (safety indicator) and LOS (efficacy indicator), respectively. Further details regarding the definition used for the primary and secondary outcomes are reported in a Supplementary file (S_Method). 


\section{Geometries of the network and risk of bias within the individual study}

The network geometries were obtained using nodes and edges to represent arms and trials, respectively. The nodes' sizes and the thicknesses of the edges in network graphs typically represent the amounts of respective evidence for specific nodes and comparisons [7]. Each network geometry was evaluated to recognize the presence of the common nodes. The absence of a common node precluded the analysis in the network for the arms. For all the endpoints, the contribution of each direct and mixed comparison was summarized using a matrix. The columns and rows indicated the direct and network estimates, respectively. The risk of bias within the individual studies was evaluated using a revised tool to assess randomized trials' bias (RoB2, latest version on July 9, 2019) [11].

\section{Summary measurements and methods of the analysis}

The treatment effect was described as the surface under the cumulative ranking (SUCRA) curves and mean ranks. These values were calculated, starting from relative rank probability. The SUCRA value represents the odds, without uncertainty, that each intervention would be the best option. The mean rank represents the treatment position in a ranking in which the ideal treatment has a mean rank equal to 1 [12, 13]. Moreover, the SUCRA values of the primary endpoint and the LOS were plotted in a safety/efficacy diagram to assess the best choice [14]. Besides, the network estimates were also reported in a pairwise form (head to head comparison) using odds ratios (ORs) or standardized mean differences (SMDs) for dichotomous outcomes and continuous variables, respectively. The measurements were expressed using $95 \%$ confidence intervals (CIs) and $95 \%$ predicting intervals (PrIs). The network meta-analysis was based on the frequentist approach, and the ranking probabilities were obtained by drawing the coefficients 1000 times. The linear predictor for each study was evaluated for each draw, and the largest linear predictor was noted [15].

\section{Inconsistency, risk of bias across the studies, and meta-regression analysis}

The network's reliability was assessed by evaluating the inconsistency [16] with local and global approaches. The local approach was measured within the closed loop and reported as the ratio of two odds ratios (RoR) or the absolute difference between the direct and indirect estimation (IF) with a $95 \%$ confidence interval. When a closed loop was absent, the inconsistency was evaluated with the global approach, using the Chi-square. The heterogeneity was measured and reported as tau $(\tau)$ [17]. When the $\tau$ value was $>0.5$, a multivariate meta-regression analysis was made to identify the factors having a significant effect $(P$ value $<0.05)$. The covariates analyzed were country, design (single-institution or multicenter study), mean age, body mass index (BMI), tumor size, rate of malignant tumors, phaeochromocytomas, side of adrenalectomy, type of healthcare system, and quality of the study. Publication/reporting bias was tested using Egger's and Begg's test [18] $(P$ value $<0.05)$. When a publication bias was discovered, a "trim and fill" adjustment was made [19].

\section{Results}

\section{Studies selected}

The results of the systematic research of the literature following the PRISMA statement are reported in Fig. 1. The search identified 9787 records (3508 from PubMed, 4643 from Scopus, and 1636 from ISI Web of Science). Five thousand one hundred forty-four papers were excluded because they were duplicate publications according to the title. Of the remaining 4643 references, 3985 were excluded since, according to the title and abstract, they were not pertinent to our field of study. Six hundred fifty-eight full-text articles were reviewed. Of these, 649 were excluded: 522 were reviews, 125 were non-randomized studies, one was a randomized study without extractable data, one contained duplicated data, and one included patients with open approach. Finally, eight studies [20-27] were eligible for the analysis. On reviewing the data extraction, there was $100 \%$ agreement between the two reviewers. The characteristics of the selected studies are summarized in Table 1.

\section{Study characteristics, network structures, and geometries}

Eight studies involving a total of 359 patients with adrenal lesions were included. The meta-analytical population had a mean age of $50 \pm 10$ years, a male-female ratio of 0.4 . The mean size of the lesions was $3.4 \pm 0.9 \mathrm{~cm}$. The distribution of the interventions was the following: 175 (48.7\%) in the TPLA arm; 55 (15.3\%) in the RPLA arm; 10 (2.8\%) in the Ro-TPLA arm; 25 (7\%) in the TPAA arm; 20 (5.6\%) in the SILS-LA arm; and 74 (20.6\%) in the RPA arm (Supplementary Table 1). The primary endpoint's network geometry is shown in Fig. 2; there were six arms, five direct comparisons, only one common node (TPLA), and no quadratic or triangular loops. Several direct comparisons were lacking because all studies included the TPLA arm. Supplementary Fig. 1- panel A reports the contribution of each comparison. It should be noted that all direct comparisons contributed 


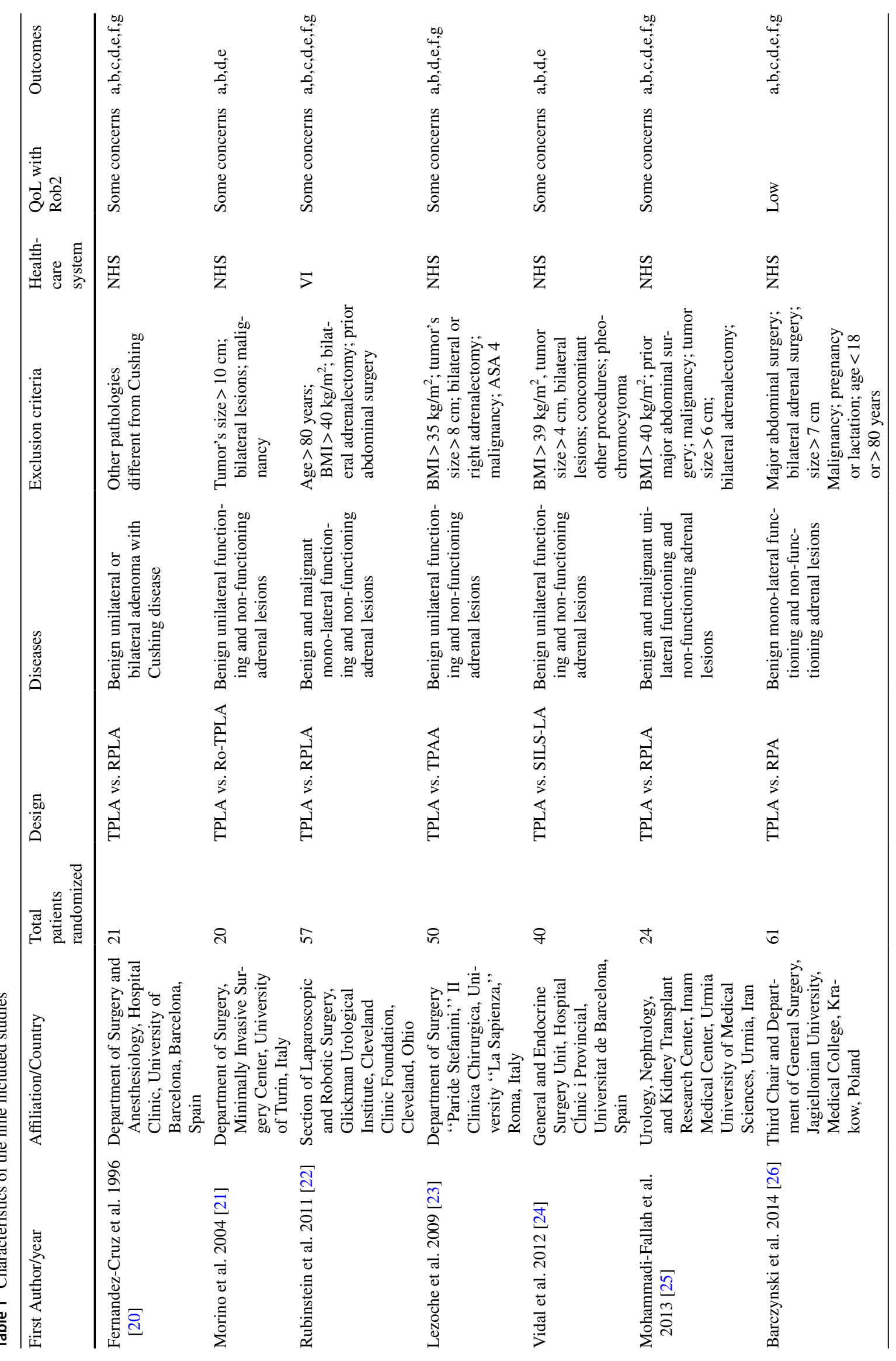




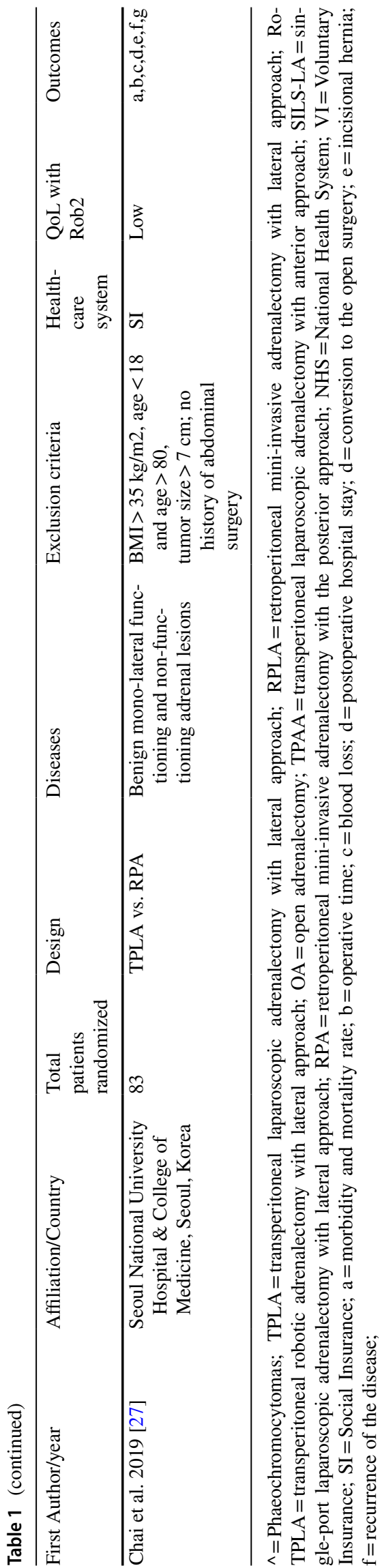

similarly to the network estimates. The operative time, LOS, and the conversion rate have a network geometry similar to the primary endpoint. On the contrary, blood loss, incisional hernia, and recurrence rate were reported in supplementary Figs. 2- panel A and B. No closed loops were found for the secondary endpoints. An exhaustive representation of contribution plots for all secondary endpoints is reported in Supplementary Figs. 2 (Panel B-G).

\section{Risk of bias within studies}

The quality of the included studies is summarized in Table 1 and is detailedly reported in Supplementary Fig. 3: six studies [20-25] have "some concerns" in the randomization process, two studies $[20,21]$ in the "intended intervention," and six [20-25] in the "reported result." No study with a high risk of bias was included, but seven studies have some concerns in the overall evaluation. The matching for age, gender, tumor size, BMI, and right adrenalectomy is reported in Supplementary Table 2.

\section{Synthesis of results}

The SUCRA and the mean rank values for the available intervention arm are shown in Table 2, while the relative ranking probabilities are displayed in Supplementary Table 3. The "head to head" comparisons for all endpoints are reported in Supplementary Fig. 4 (panel A-G).

\section{Primary endpoints}

Regarding the primary endpoint, none of the approaches was near to an ideal approach. RPLA and RPA were the approaches having the highest probability of being the safest (69.6\% and 63.0\%, respectively). On average, RPLA and RPA have a mean rank near 3. TPAA, Ro-TPLA, and SILSLA TPLA were the second choices (mean rank near 4) with SUCRA values of $45.2 \%, 43.4 \%, 43.0 \%, 38.5 \%$, respectively.

\section{Secondary endpoints}

Regarding the operative time, the RPA was the approach with the highest probability of being the fastest (SUCRA 87.5\%; mean rank 1.6). The second choices were TPAA with a SUCRA value of 54.5 (mean rank 3.3). The approaches with the lowest probability of being the fastest procedures were the TPLA, RPLA, Ro-TPLA, and SILS-LA with SUCRA values of $45.1 \%, 45.1 \%, 35.7 \%, 32.1 \%$, respectively.

Data about blood loss are lacking or not extractable for Ro-TPLA, TPAA, or SILS-LA. The approach having the highest probability of being those with the lowest blood loss was RPA (SUCRA 94.7\%; mean rank 1.1). TPLA and RPLA were the second choices (mean rank around 3) with 


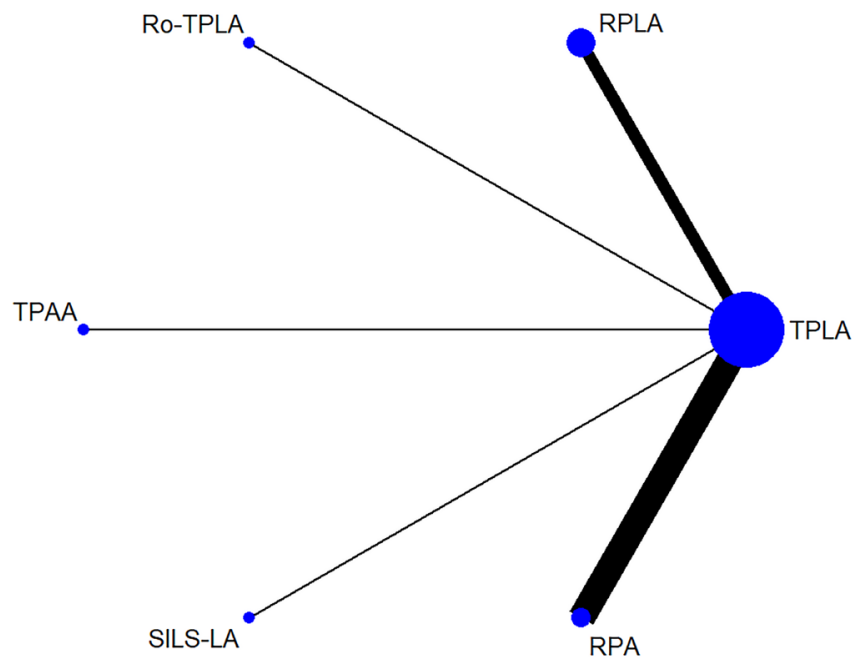

Fig. 2 Network geometry for the primary endpoint (mortality or major complications). The network geometry graphically describes the direct comparisons available in the literature. In the figure, the blue nodes represent the interventions compared, while the edges represent the direct comparisons available (comparisons evaluated in at least one study) between pairs of interventions. TPLA $=$ Trans- peritoneal laparoscopic lateral adrenalectomy; RPLA $=$ retroperitoneal mini-invasive lateral adrenalectomy; Ro-TPLA =transperitoneal laparoscopic lateral adrenalectomy with robotic approach; TPAA = transperitoneal laparoscopic anterior adrenalectomy (TPAA); SILS-LA = single-incision laparoscopic adrenalectomy; RPA = retroperitoneal mini-invasive posterior adrenalectomy

Table 2 Surface under cumulative ranking area (SUCRA) values and mean rank for all outcomes. The SUCRA values express the percentage of each approach's safety or efficacy relative to an imaginary approach, which was always the best without uncertainty

\begin{tabular}{|c|c|c|c|c|c|c|c|c|c|c|c|c|c|}
\hline \multirow[t]{3}{*}{ Outcomes of interest } & \multirow[t]{3}{*}{ Studies } & \multicolumn{12}{|c|}{ SUCRA (\%) and Rank (mean) for arm } \\
\hline & & \multicolumn{2}{|l|}{ TPLA } & \multicolumn{2}{|l|}{ RPLA } & \multicolumn{2}{|c|}{ Ro-TPLA } & \multicolumn{2}{|l|}{ TPAA } & \multicolumn{2}{|c|}{ SILS-LA } & \multicolumn{2}{|l|}{ RPA } \\
\hline & & SUCRA & Rank & SUCRA & Rank & SUCRA & Rank & SUCRA & Rank & SUCRA & Rank & SUCRA & Rank \\
\hline Composite endpoint ${ }^{\wedge}$ & 8 & 38.5 & 4.2 & 69.6 & 2.5 & 43.4 & 3.8 & 45.2 & 3.7 & 43.0 & 3.9 & 63.0 & 2.9 \\
\hline Operative time & 8 & 45.1 & 3.7 & 45.1 & 3.7 & 35.7 & 4.2 & 54.5 & 3.3 & 32.1 & 4.4 & 87.5 & 1.6 \\
\hline Blood loss & 5 & 28.6 & 2.4 & 26.6 & 2.5 & $*$ & $*$ & $*$ & $*$ & $*$ & $*$ & 94.7 & 1.1 \\
\hline LOS & 8 & 42.3 & 3.9 & 44.9 & 3.8 & 44.8 & 3.8 & 46.9 & 3.7 & 44.2 & 3.8 & 76.9 & 2.2 \\
\hline Conversion & 8 & 52.2 & 3.4 & 45.8 & 3.7 & 51.1 & 3.4 & 50.1 & 3.5 & 49.9 & 3.5 & 51.0 & 3.5 \\
\hline Incisional hernia & 6 & 26.8 & 3.2 & 59.2 & 2.2 & $*$ & $*$ & 35.3 & 2.9 & $*$ & $*$ & 78.6 & 1.6 \\
\hline Disease Recurrence & 6 & 52.2 & 2.4 & 41.8 & 2.7 & $*$ & $*$ & 52.8 & 2.4 & $*$ & $*$ & 53.2 & 2.4 \\
\hline
\end{tabular}

SUCRA = the surface under the cumulative ranking curve; LOS = length of postoperative stay; TPLA = transperitoneal laparoscopic adrenalectomy with lateral approach; RPLA = retroperitoneal mini-invasive adrenalectomy with lateral approach; Ro-TPLA=transperitoneal robotic adrenalectomy with lateral approach; TPAA = transperitoneal laparoscopic adrenalectomy with anterior approach; SILS-LA = single-port laparoscopic adrenalectomy with lateral approach; $\mathrm{RPA}=$ retroperitoneal mini-invasive adrenalectomy with the posterior approach; ${ }^{\wedge}=$ morbidity and mortality rate; $*$ data not available for this arm

SUCRA values of $28.6 \%$ and $26.6 \%$. Considering the LOS, the best approach was the RPA (SUCRA 76.9\%; mean rank 2.2). TPLA, RPLA, Ro-TPLA, TPAA, and SILS-LA have equal chances in guaranteeing the shortest hospitalization (mean rank near 4, and SUCRA values around 40\%). All minimally invasive approaches have similar chances of avoiding a conversion in laparotomy (SUCRA values around 50\% and mean rank around 3.5). Incisional hernia 
and disease recurrence were not available for Ro-TPLA and SILS-LA. The approaches with the highest chances to avoid an incisional hernia were the RPA (SUCRA 78.6\%; mean rank 1.6) and RPLA (SUCRA 59.2\%; mean rank $2.2)$. The second choices were TPAA and TPLA, with SUCRA values of $35.3 \%$ and $26.8 \%$. All approaches have similar chances of avoiding a disease recurrence (SUCRA values around 50\% and mean rank around 3).

\section{Safety/efficacy combination}

The combination of safety/efficacy is plotted in Fig. 3. The RPA arm was better than the other approaches, having the highest probability of being both safe and efficacious (Cophenetic correlation coefficient $\mathrm{c}=0.95$, maximum clustering gain $=461$, and an optimal number of clusters $=4)$. The second and third choice combining high safety and efficacy were the RPLA and TPAA. The RoTPLA, SILS-LA, and TPLA approaches had the lowest probability of being the safest and the most efficacious.

\section{Inconsistency, heterogeneity, and publication bias}

Inconsistency and heterogeneity for all outcomes are shown in Table 3. For all the endpoints, no source inconsistency was found within the networks. Heterogeneity was very low ( $\tau$ value $<0.1$ ) for the primary endpoint, conversion, incisional hernia, and disease recurrence rate. On the contrary, heterogeneity was high for operative time, blood loss, and LOS. The funnel plots are reported in Supplementary Fig. 5; panels A-G. The Begg and Egger test was made only for TPLA vs. RPLA because the other direct comparisons did not present sufficient observation. Egger's tests showed a significant small effect for incisional hernia and disease recurrence $(P=0.005$ and $P=0.001)$. However, the "trim and fill" adjustment did not show significant OR changes.

\section{Meta-regression analysis}

Meta-regression analysis was carried out for the LOS operative time and blood loss, and is reported in Table 4 and supplementary $\mathbf{a}$, and $\mathbf{b}$. None of the covariates explained the relatively high heterogeneity ( $P>0.05$ for all covariates).

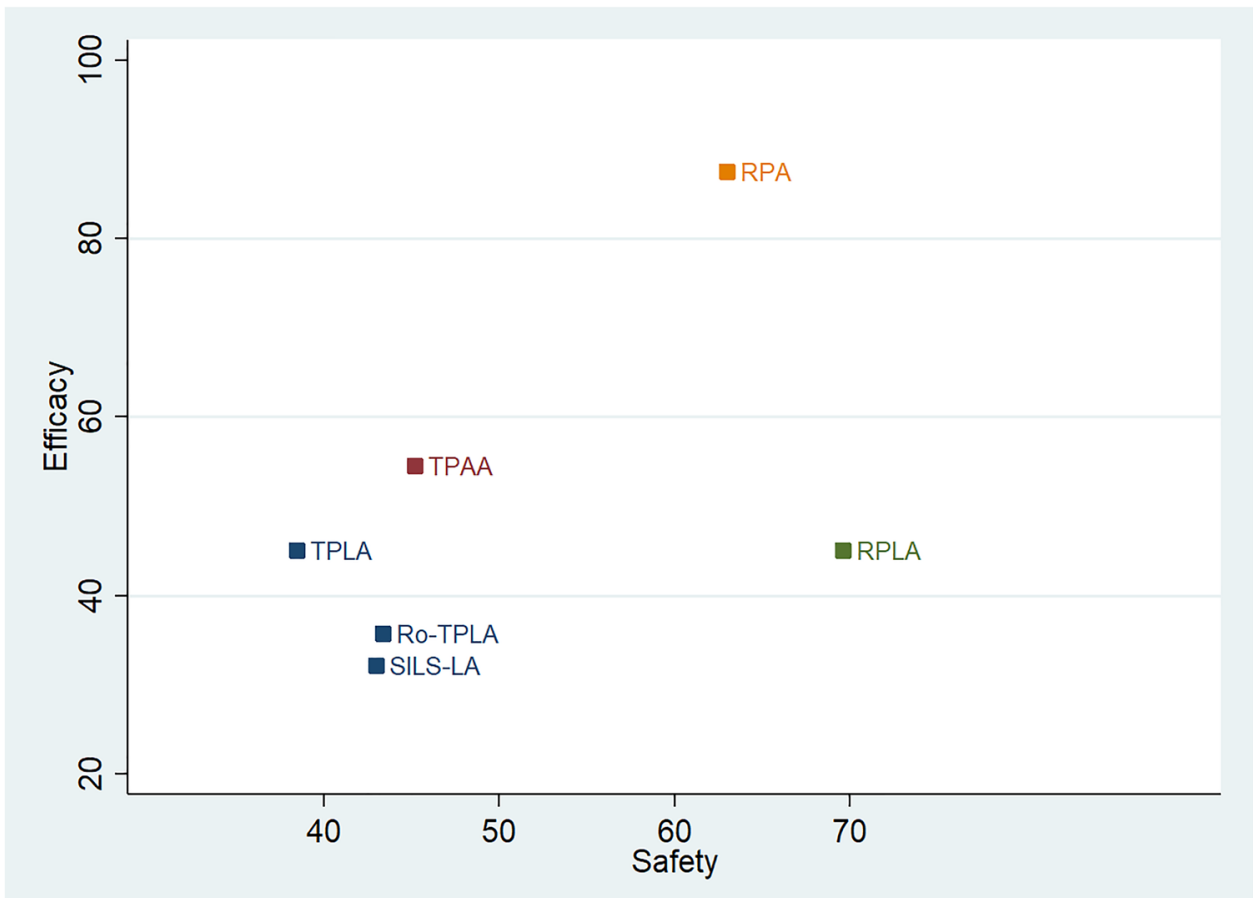

Fig. 3 Safety/efficacy combination of all the approaches available for treating adrenal neoplasms. In the figure, the primary endpoint (safety indicator) is combined with a secondary endpoint (surrogate parameter of efficacy). Cluster rank combined the surface under the cumulative ranking curve (SUCRA) values of the composite endpoint and length of stay. On the y-axis, the SUCRA values correspond to the probability in percentages that each approach was the safest. On the $\mathrm{x}$-axis, the SUCRA values correspond to the probability in per- centages that each approach was most efficacious. Different colors identify the different clusters. TPLA $=$ Transperitoneal laparoscopic lateral adrenalectomy; RPLA = retroperitoneal mini-invasive lateral adrenalectomy; Ro-TPLA $=$ transperitoneal laparoscopic lateral adrenalectomy with robotic approach; TPAA $=$ transperitoneal laparoscopic anterior adrenalectomy; SILS-LA = single-incision laparoscopic adrenalectomy; RPA = retroperitoneal mini-invasive posterior adrenalectomy 
Table 3 Inconsistency, heterogeneity, and publication bias

\begin{tabular}{|c|c|c|c|c|c|c|c|c|c|}
\hline \multirow[t]{3}{*}{ Outcomes of interest } & \multicolumn{4}{|c|}{ Inconsistency } & \multirow[t]{3}{*}{$\tau$ value } & \multirow{2}{*}{\multicolumn{2}{|c|}{$\begin{array}{l}\text { Publication } \\
\text { bias^ }^{\wedge} \\
\text { TPLA vs. RPLA }\end{array}$}} & \multirow{2}{*}{\multicolumn{2}{|c|}{$\begin{array}{l}\text { OR }(95 \mathrm{CI}) \text { and Adj-OR }(95 \mathrm{CI})^{\wedge} \\
{\text { TPLA vs. } \text { RPLA }^{\wedge}}\end{array}$}} \\
\hline & \multicolumn{2}{|l|}{ Global } & \multicolumn{2}{|l|}{ Local } & & & & & \\
\hline & Chi-square & $P$ value & Loop & RoR; $P$ value & & Begg & Egger & & \\
\hline Composite endpoint & 1.34 & 0.246 & No & $*$ & $<0.1$ & 1.000 & 0.813 & $0.36(0.08-1.93)$ & $0.36(0.08-1.93)$ \\
\hline Operative time & 2.43 & 0.119 & No & $*$ & $>1$ & $*$ & $*$ & $*$ & $*$ \\
\hline Blood loss & 3.41 & 0.065 & No & $*$ & $>1$ & $*$ & $*$ & $*$ & $*$ \\
\hline$L O S$ & 1.14 & 0.286 & No & $*$ & 0.9 & $*$ & $*$ & $*$ & $*$ \\
\hline Conversion & 0.01 & 0.998 & No & $*$ & $<0.1$ & 1.000 & 0.910 & $1.25(0.19-8.30)$ & $1.25(0.19-8.30)$ \\
\hline Incisional hernia & 1.91 & 0.167 & No & $*$ & $<0.1$ & 1.000 & 0.005 & $0.39(0.05-3.17)$ & $0.39(0.05-3.17)$ \\
\hline Disease recurrence & 0.01 & 0.998 & No & $*$ & $<0.1$ & 0.296 & 0.001 & $1.40(0.17-11.80)$ & $1.40(0.17-11.80$ \\
\hline
\end{tabular}

TPLA $=$ transperitoneal laparoscopic adrenalectomy with lateral approach; RPLA=retroperitoneal mini-invasive adrenalectomy with lateral approach; RoR $=$ ratio of odds ratios; $\mathrm{LOS}=$ length of postoperative stay; $*=$ not computable; $\mathrm{OR}=$ odds ratio; Adj-OR $=$ adjusted odds ratio based on trim and fill approach; $95 \mathrm{CI}=$ confidence interval at $95 \%{ }^{\wedge}=$ publication bias was analyzed only for TPLA vs. RPLA comparison because the other direct comparisons did not contain a sufficient number of observations

Table 4 Meta-regression analysis for the length of stay

\begin{tabular}{|c|c|c|c|c|c|c|c|}
\hline \multirow[t]{2}{*}{ Covariates } & \multicolumn{7}{|c|}{$\begin{array}{l}\text { Postoperative stay } \\
\text { SMD (95 CI; P value) }\end{array}$} \\
\hline & $\mathrm{OA}$ & TPLA & RPLA & Ro-TPLA & TPAA & SILS-LA & $R P A$ \\
\hline Country (Western vs. Eastern) & Referent & $3( \pm 153 ; 0.984)$ & $1.6( \pm 153 ; 0.991)$ & $*$ & $*$ & $*$ & $4.1( \pm 153 ; 0.979)$ \\
\hline Proportion of male patients (RR) & Referent & $15.1( \pm 200.2 ; 0.940)$ & $12.8( \pm 200.2 ; 0.949)$ & $*$ & $*$ & $*$ & $0.9( \pm 200.5 ; 0.997)$ \\
\hline Difference in age (SMD) & Referent & $-11.3( \pm 110.9 ; 0.919)$ & $-22.1( \pm 111 ; 0.842)$ & $*$ & $*$ & $*$ & $-44.6( \pm 115.3 ; 0.699)$ \\
\hline Difference in BMI (SMD) & Referent & $-3.2( \pm 30 ; 0.917)$ & $-1.8( \pm 30.6 ; 0.953)$ & $*$ & $*$ & $*$ & * \\
\hline Difference in tumor size (SMD) & Referent & $12.1( \pm 366.1 ; 0.974)$ & $16.8( \pm 366.1 ; 0.963)$ & $*$ & $*$ & $*$ & $2.1( \pm 366.2 ; 0.995)$ \\
\hline Malignant tumor (No vs. Yes) & Referent & $2.9( \pm 31.8 ; 0.925)$ & $3.1( \pm 31.8 ; 0.997)$ & $*$ & $*$ & $*$ & $*$ \\
\hline Phaeochromocytomas (No vs. Yes) & Referent & $-2.8( \pm 152 ; 0.985)$ & $-38.4( \pm 3067 ; 0.990)$ & $*$ & $*$ & $*$ & $*$ \\
\hline Bilateral tumor (No vs. Yes) & Referent & $2.4( \pm 316.3 ; 0.998)$ & $3.8( \pm 316 ; 0.990)$ & $*$ & $*$ & $*$ & $*$ \\
\hline $\begin{array}{l}\text { Proportion of right adrenalectomy } \\
\text { (RR) }\end{array}$ & Referent & $*$ & $*$ & $*$ & $*$ & $*$ & $*$ \\
\hline $\begin{array}{l}\text { Healthcare system (National vs. } \\
\text { Insurance-based) }\end{array}$ & Referent & $2.9( \pm 31.3 ; 0.924)$ & $3.1( \pm 31.4 ; 0.924)$ & $*$ & $*$ & $*$ & $3.9( \pm 31.4 ; 0.899)$ \\
\hline $\begin{array}{l}\text { Study quality (low risk vs some } \\
\text { concerns) }\end{array}$ & Referent & $-3.2( \pm 123.1 ; 0.979)$ & $*$ & $*$ & $*$ & $*$ & $*$ \\
\hline
\end{tabular}

$\mathrm{RR}=$ risk ratio $\mathrm{SMD}=$ standard mean difference; $\mathrm{TPLA}=$ transperitoneal laparoscopic adrenalectomy with lateral approach; RPLA $=$ retroperitoneal mini-invasive adrenalectomy with lateral approach; Ro-TPLA = transperitoneal robotic adrenalectomy with lateral approach; OA = open adrenalectomy; TPAA = transperitoneal laparoscopic adrenalectomy with anterior approach; SILS-LA = single-port laparoscopic adrenalectomy with lateral approach; RPA = retroperitoneal mini-invasive adrenalectomy with the posterior approach; SMD=standardized mean difference; $*=$ not computable

\section{Discussion}

Our study suggests that RPA and RPLA can be considered the best choices for patients who are candidate for minimally invasive adrenalectomy. These results were sustained by a meta-analysis that included only RCTs for the first time. Moreover, we analyzed the studies in a network format. Besides, even if several meta-analysis [28-46] has been produced in the last 20 years, all were affected by two major limitations: a) the bias due to non-randomized studies included; b) the difficulty to obtain clear evidence about the best technique due to the multi-arm setting. Our systematic review included only 6 phase II/III RCTs involving 359 patients because all high-risk studies were discharged.

The meta-analytic population well represents the patient candidate for minimally invasive adrenalectomy, with a 
mean age of 50 years and a mean size of the lesions near $3 \mathrm{~cm}$. Our systematic review confirmed that the most studied minimally invasive techniques were the transperitoneal approaches (TPLA in particular), representing almost 50\% of the entire cohort, followed by the retroperitoneal ones (RPA and RPLA, near 30\% of the procedures). Due to their limited popularity, the robotic or single-incision approaches are rarely studied in high-quality studies (RCTs). These data confirmed those of a recent large multicentric study of Pavan et al. [47], in which the conventional laparoscopic approach resulted in the preferred way ( $46 \%$ of cases) compared to robotic or single-incision approaches.

Moreover, despite the popularity of TPLA, our results suggest that RPA and RPLA seem to be the minimally invasive approaches that guarantee the lowest morbidity rate. The remaining techniques have a very similar chance of being the best choice (near 40-50\%). This datum is robust because, despite a certain degree of heterogeneity among the included studies (exclusion criteria and type of disease), the evaluation of the network's coherence did not demonstrate a source of inconsistency.

These results were different from those reported by Heger et al. [35], in which the inclusion of non-randomized studies prevents seeing the differences between the transperitoneal and retroperitoneal approaches. Moreover, the large difference in morbidity observed by Heger et al. [35] in favor of the robotic approach disappeared, and this phenomenon was probably due to the selection bias of non-randomized studies. Other advantages of the retroperitoneal approach were observed for some secondary endpoints (OP, blood loss, LOS, and incisional hernia).

On the contrary, all minimally invasive techniques were similar as concern conversion rate and disease recurrence. Once again, the high quality of included studies in our meta-analysis provided us different results from Heger et al. [35] First, the OP was considered the Achilles' heel of the minimally invasive approach, but our meta-analysis did not confirm this limitation. Even if some approaches, such as robotic or SILS-based, could result slightly slower than others, these differences were minimal. Indeed, comparative studies' randomized design probably minimized the differences due to the selection bias, incomplete learning curve, or group imbalance. The only approach superior to the other seems to be the RPA. The reason for that was well underlined by Barczynski et al. [26]: the posterior technique offers direct access to the surgical site, making the operation very fast and easy. The lateral approaches (retroperitoneoscopic or transperitoneal) imply a less direct approach due to the necessity to dissect the adrenal gland from the kidney. About blood loss, our data confirmed the superiority of any minimally invasive approaches compared with the laparotomic one and established a clear hierarchy among the minimally invasive options in favor of retroperitoneal access.
This could be explained considering that the adrenal glands are retroperitoneal, and the transperitoneal approach requires a large mobilization of the intra-peritoneal organs. Considering the LOS and incisional hernia, our study confirmed that all minimally invasive approaches were superior to the open, but this difference was more remarkable between the open and RPA approach. The explanation of these data could be found in the not opening of the peritoneal cavity using the retroperitoneal approach. All results seem to be robust because, despite a certain degree of heterogeneity among the included studies (exclusion criteria and type of disease), the evaluation of the network's coherence did not demonstrate the presence of inconsistency. Significant heterogeneity was found for some secondary endpoints such as LOS, blood loss, and operative time. The meta-regression did not explain the reasons for heterogeneity. However, several nonmeasurable or non-extractable factors could influence these parameters, such as the modality of blood loss or operative time measurement or learning curve.

This study had some limitations. Firstly, the number of included patients was smaller than other meta-analyses available [28-46]. Nonetheless, the present study remains the only meta-analysis including exclusively RCTs, providing high-quality evidence about minimally invasive adrenalectomy.

Secondly, the learning curve differences among the included studies could influence the outcomes related to the surgeon's skills. Indeed, it is well known that OP could vary for many factors, including the surgeon's experience or patient characteristics [48]. Thus, the results about OP should be interpreted with caution even if the meta-regression analysis did not discover heterogeneity sources. Thirdly, the included studies were conducted in different countries with different healthcare systems. These differences could influence some critical outcomes, such as the LOS. Indeed, the LOS presented a certain degree of heterogeneity, even if the meta-regression analysis did not show any factor able to influence the LOS results. Finally, despite the lack of inconsistency, the eight studies included have some differences in the type of disease, inclusion, and exclusion criteria.

In conclusion, our study demonstrated with high-quality evidence that RPA could represent the best option for patients who are candidate to minimally invasive adrenalectomy. RPLA was a valid alternative to the posterior approach. Even when robotic platform was employed, the transperitoneal approach was a sub-optimal choice. However, these approaches should not be abandoned because a tailored approach could be required in particular settings such as paraganglioma, large adrenal masses, or hereditary syndromes.

Supplementary Information The online version contains supplementary material available at https://doi.org/10.1007/s00423-022-02431-w. 
Authors' contribution Alberici L, Ricci C conceptualized and designed the study and drafted the manuscript. Ingaldi D, Vicennati V, Di Dalmazi G, Selva S acquired the data. Ricci C, Pagotto U analyzed and interpreted the data. Casadei R, Minni F helped in critical revision.

\section{Declarations}

Conflicts of interests/Competing interests All authors disclose financial and non-financial interests that are directly or indirectly related to the work submitted for publication. Finally, all authors disclose any conflict of interests.

Open Access This article is licensed under a Creative Commons Attribution 4.0 International License, which permits use, sharing, adaptation, distribution and reproduction in any medium or format, as long as you give appropriate credit to the original author(s) and the source, provide a link to the Creative Commons licence, and indicate if changes were made. The images or other third party material in this article are included in the article's Creative Commons licence, unless indicated otherwise in a credit line to the material. If material is not included in the article's Creative Commons licence and your intended use is not permitted by statutory regulation or exceeds the permitted use, you will need to obtain permission directly from the copyright holder. To view a copy of this licence, visit http://creativecommons.org/licenses/by/4.0/.

\section{References}

1. Gagner M, Lacroix A, Bolté E (1992) Laparoscopic adrenalectomy in Cushing's syndrome and pheochromocytoma. N Engl J Med 327(14):1033

2. Lezoche E, Guerrieri M, Paganini AM et al (2000) Laparoscopic adrenalectomy by the anterior transperitoneal approach: results of 108 operations in unselected cases. Surg Endosc 14(10):920-925

3. Mercan S, Seven R, Ozarmagan S et al (1995) Endoscopic retroperitoneal adrenalectomy. Surgery 118(6):1071-5 (discussion 1075-6)

4. Walz MK, Alesina PF, Wenger FA et al (2006) Retroperitoneoscopic adrenalectomy-results of 560 procedures in 520 patients. Surgery 140(6):943-8 (discussion 948-50)

5. Bonjer HJ, Sorm V, Berends FJ, Kazemier G, Steyerberg EW, de Herder WW, Bruining HA (2000) Endoscopic retroperitoneal adrenalectomy: lessons learned from 111 consecutive cases. Ann Surg 232(6):796-803

6. Higgins JPT, Green S (2011) Cochrane Handbook for Systematic Reviews of Interventions Version 5.1.0 [updated March 2011]. The Cochrane Collaboration. Available from http://handbook. cochrane.org. Accessed Jul 32019

7. Hutton B, Salanti G, Caldwell DM et al (2015) The PRISMA extension statement for reporting of systematic reviews incorporating network meta-analyses of health care interventions: checklist and explanations. Ann Intern Med 11:777-784

8. Eriksen MB, Frandsen TF (2018) The impact of patient, intervention, comparison, outcome (PICO) as a search strategy tool on literature search quality: a systematic review. J Med Libr Assoc 106:420-421

9. Ricci C, Pagano N, Taffurelli G et al (2018) Comparison of Efficacy and Safety of 4 Combinations of Laparoscopic and Intraoperative Techniques for Management of Gallstone Disease With Biliary Duct Calculi: a Systematic Review and Network Metaanalysis. JAMA Surg 153(7):e181167

10. Ricci C, Pagano N, Ingaldi C, et al (2020) Treatment for Infected Pancreatic Necrosis Should be Delayed, Possibly Avoiding an
Open Surgical Approach: A Systematic Review and Network Meta-analysis. Ann Surg. [Epub ahead of print]

11. Sterne JAC, Savović J, Page MJ, et al (2022) RoB 2: a revised tool for assessing risk of bias in randomized trials. BMJ

12. Caldwell DM, Ades AE, Higgins JP (2005) Simultaneous comparison of multiple treatments: combining direct and indirect evidence. BMJ 331:897-900

13. Mills EJ, Ioannidis JP, Thorlund K et al (2012) How to use an article reporting a multiple treatment comparison meta-analysis. JAMA 308:1246-1253

14. Chaimani A, Higgins JP, Mavridis D et al (2013) Graphical tools for network meta-analysis in STATA. PLoS One 8:e76654

15. Salanti G, Ades AE, Ioannidis JP (2011) Graphical methods and numerical summaries for presenting results from multiple-treatment meta-analysis: an overview and tutorial. J Clin Epidemiol 64:163-171

16. Bucher HC, Guyatt GH, Griffith LE et al (1997) The results of direct and indirect treatment comparisons in meta-analysis of randomized controlled trials. J Clin Epidemiol 50:683-691

17. Turner RM, Davey J, Clarke MJ et al (2012) Predicting the extent of heterogeneity in meta-analysis, using empirical data from the Cochrane Database of Systematic Reviews. Int J Epidemiol 41:818-827

18. Egger M, Davey Smith G et al (1997) Bias in meta-analysis detected by a simple, graphical test. BMJ 315:629-634

19. Gjerdevik M, Heuch I (2014) Improving the error rates of the Begg and Mazumdar test for publication bias in fixed effects metaanalysis. BMC Med Res Methodol 22:109

20. Fernández-Cruz L, Saenz A, Benarroch G, Astudillo E, Taura P, Sabater L (1996) Laparoscopic unilateral and bilateral adrenalectomy for Cushing's syndrome Transperitoneal and retroperitoneal approaches. Ann Surg 224:727-736

21. Morino M, Benincà G, Giraudo G et al (2004) Robot-assisted vs. laparoscopic adrenalectomy: a prospective randomized controlled trial. Surg Endosc 18:1742-1746

22. Rubinstein M, Gill IS, Aron M et al (2005) Prospective, randomized comparison of transperitoneal versus retroperitoneal laparoscopic adrenalectomy. J Urol 174:442-445

23. Lezoche E, Guerrieri M, Crosta F et al (2008) Flank approach versus anterior sub-mesocolic access in left laparoscopic adrenalectomy: a prospective randomized study. Surg Endosc 22:2373-2378

24. Vidal Ó, Astudillo E, Valentini M et al (2012) Single-incision transperitoneal laparoscopic left adrenalectomy. World J Surg 36:1395-1399

25. Mohammadi-Fallah MR, Mehdizadeh A, Badalzadeh A et al (2013) Comparison of transperitoneal versus retroperitoneal laparoscopic adrenalectomy in a prospective randomized study. J Laparoendosc Adv Surg Tech A 23:362-366

26. Barczyński M, Konturek A, Nowak W (2014) Randomized clinical trial of posterior retroperitoneoscopic adrenalectomy versus lateral transperitoneal laparoscopic adrenalectomy with a 5-year follow-up. Ann Surg 260:740-747

27. Chai YJ, Yu HW, Song RY et al (2019) Lateral Transperitoneal Adrenalectomy Versus Posterior Retroperitoneoscopic Adrenalectomy for Benign Adrenal Gland Disease: Randomized Controlled Trial at a Single Tertiary Medical Center. Ann Surg 269:842-848

28. Economopoulos KP, Mylonas KS, Stamou AA et al (2017) Laparoscopic versus robotic adrenalectomy: a comprehensive metaanalysis. Int J Surg 38:95-104

29. Wu S, Lai H, Zhao J et al (2016) Laparoendoscopic Single-site Adrenalectomy versus Conventional Laparoscopic Adrenalectomy: an Updated Meta Analysis. Urol J 16(13):2590-2598

30. Jiang YL, Qian LJ, Li Z et al (2020) Comparison of the retroperitoneal versus Transperitoneal laparoscopic Adrenalectomy 
perioperative outcomes and safety for Pheochromocytoma: a meta-analysis. BMC Surg 13(20):12

31. Danwang C, Agbor VN, Bigna JJ (2020) Obesity and postoperative outcomes of the patients with laparoscopic adrenalectomy: a systematic review and meta-analysis. BMC Surg 31(20):194

32. Li J, Wang Y, Chang X, Han Z (2020) Laparoscopic adrenalectomy (LA) vs open adrenalectomy (OA) for pheochromocytoma (PHEO): a systematic review and meta-analysis. Eur J Surg Oncol 46:991-998

33. Tang K, Li H, Xia D et al (2015) Robot-assisted versus laparoscopic adrenalectomy: a systematic review and meta-analysis. J Laparoendosc Adv Surg Tech A 25:187-195

34. Brandao LF, Autorino R, Laydner H et al (2014) Robotic versus laparoscopic adrenalectomy: a systematic review and meta-analysis. Eur Urol 65:1154-1161

35. Heger P, Probst P, Hüttner FJ et al (2017) Evaluation of Open and Minimally Invasive Adrenalectomy: a Systematic Review and Network Meta-analysis. World J Surg 41:2746-2757

36. Nigri G, Rosman AS, Petrucciani N et al (2013) Meta-analysis of trials comparing laparoscopic transperitoneal and retroperitoneal adrenalectomy. Surgery 153:111-119

37. Hu Q, Gou Y, Sun C et al (2013) A systematic review and metaanalysis of current evidence comparing laparoendoscopic singlesite adrenalectomy and conventional laparoscopic adrenalectomy. J Endourol 27:676-683

38. Chen W, Li F, Chen D et al (2013) Retroperitoneal versus transperitoneal laparoscopic adrenalectomy in adrenal tumor: a metaanalysis. Surg Laparosc Endosc Percutan Tech 23:121-127

39. Constantinides VA, Christakis I, Touska P et al (2012) Systematic review and meta-analysis of retroperitoneoscopic versus laparoscopic adrenalectomy. Br J Surg 99:1639-1648

40. Wang L, Wu Z, Li M et al (2013) Laparoendoscopic singlesite adrenalectomy versus conventional laparoscopic surgery: a systematic review and meta-analysis of observational studies. J Endourol 27:743-750

41. Autorino R, Bove P, De Sio M et al (2016) Open Versus Laparoscopic Adrenalectomy for Adrenocortical Carcinoma: a Metaanalysis of Surgical and Oncological Outcomes. Ann Surg Oncol 23:1195-1202

42. Nagaraja V, Eslick GD, Edirimanne S (2015) Recurrence and functional outcomes of partial adrenalectomy: a systematic review and meta-analysis. Int J Surg 16:7-13

43. Sgourakis G, Lanitis S, Kouloura A et al (2015) Laparoscopic versus Open Adrenalectomy for Stage I/II Adrenocortical Carcinoma: Meta-Analysis of Outcomes. J Invest Surg 28:145-152

44. Carnaille B (2012) Adrenocortical carcinoma: which surgical approach? Langenbecks Arch Surg 397:195-199

45. Winoker JS, Ahlborn DT, Omidele OO et al (2018) Minimally invasive adrenal surgery: virtue or vice? Future Oncol 14:267-276

46. Han DH, Lim MS, Seo JW et al (2012) Laparoendoscopic single site adrenal surgery. Arch Esp Urol 65:336-341

47. Pavan N, Autorino R, Lee $\mathrm{H}$ et al (2016) Impact of novel techniques on minimally invasive adrenal surgery: trends and outcomes from a contemporary international large series in urology. World J Urol 34(10):1473-1479

48. Alberici L, Ricci C, Ingaldi C, et al (2020) The learning curve for the second generation of laparoscopic surgeons: lesson learned from a large series of laparoscopic adrenalectomies. Surg Endosc. [head of print]

Publisher's note Springer Nature remains neutral with regard to jurisdictional claims in published maps and institutional affiliations. 